\title{
Vibration and Dynamic Response Control of Nonuniform Composite Rotating Blades
}

\author{
Sungsoo $\mathrm{Na},{ }^{1}$ Liviu Librescu, ${ }^{2}$ Sung-Nam Rim, ${ }^{3}$ and Gwon-Chan Yoon ${ }^{1}$ \\ ${ }^{1}$ Department of Mechanical Engineering, Korea University, Seoul 136-701, South Korea \\ ${ }^{2}$ Department of Engineering Science and Mechanics, Virginia Polytechnic Institute and State University, \\ Blacksburg, VA 24061-0219, USA \\ ${ }^{3} R \& D$ Division of Hyundai-Kia Motors, 772-1, Jangduk-Dong, Hwaseong-Si, Gyeonggi-Do 445-706, South Korea
}

Received 9 August 2005; Accepted 2 September 2005

\begin{abstract}
This paper addresses the free vibration, dynamic response, and the active control of composite rotating pretwisted blades modeled as nonuniform thin-walled beams, fixed at the hub at a setting angle, and incorporating piezoelectrically induced damping capabilities. In this sense, a distributed piezoelectric actuator system activated through the application of an out-of-phase electrical current is used to suppress the dynamic response of the rotating beam subjected to a Heaviside pulse. The blade model incorporates nonclassical effects such as transverse shear, secondary warping, and rotary inertias, and includes the centrifugal and Coriolis force fields. A velocity feedback control law relating the piezoelectrically induced bending moment at the beam tip with appropriately selected kinematical response quantities is used, and the beneficial effects of its implementation upon the closed loop eigenvibration and dynamic characteristics of the blade are highlighted.
\end{abstract}

Copyright (c) 2006 Sungsoo Na et al. This is an open access article distributed under the Creative Commons Attribution License, which permits unrestricted use, distribution, and reproduction in any medium, provided the original work is properly cited.

\section{INTRODUCTION}

The study of the eigenvibration and dynamic response of rotor blades is an important prerequisite in the design of turbomachinery, helicopter, and jet engines. A good understanding of their free vibration and dynamic response is essential towards determining their fatigue life and avoiding catastrophic failures. In this connection, incorporation of composite material technology influences significantly the design of advanced rotor blades. Such a design methodology offers considerable advantages with respect to strength and weight criteria, in addition to providing adequate means of efficiently controlling static and dynamic response, via implementation of structural tailoring.

An important step toward the rational design of modern rotor blades consists of the development of analytical models that are capable of accurately predicting their dynamic response.

Moreover, in order to enhance their free vibration as well as their dynamic response, and avoid vibration-induced fatigue failure, smart materials control technology may be implemented.

Within the present study, the free vibration and dynamic response of pretwisted rotating blades of nonuniform cross- section, featuring the bending-bending transverse shear coupling and incorporating the adaptive capability referred to as induced strain actuation, are investigated. The adaptive capability is achieved through the converse piezoelectric effect that consists of the generation of localized strains in response to an applied voltage. The induced strain field produces, in turn, an adaptive change in the dynamic response characteristics of the structure.

Although this problem is of an evident practical importance, to the best of authors' knowledge no studies addressing it in a so broad way can be found in the specialized literatures. In special contexts, the free vibration of rotating/nonrotating blades modeled as thin-walled beams was considered by Song and Librescu [1, 2], Song et al. [3-5], Oh et al. [6, 7], Librescu et al. [8], Librescu and $\mathrm{Na}$ [9], $\mathrm{Na}$ and Librescu [10-13], and $\mathrm{Na}$ et al. [14].

\section{CONFIGURATION OF THE BLADE}

Figures 1 and 2 show the geometrical configuration of the tapered rotating blade and of piezoactuators, respectively.

The blade cross-section of a biconvex profile experiences a linear distribution along the beam span, in both the horizontal and vertical planes. In this sense, the chord $c(\eta)$ and 


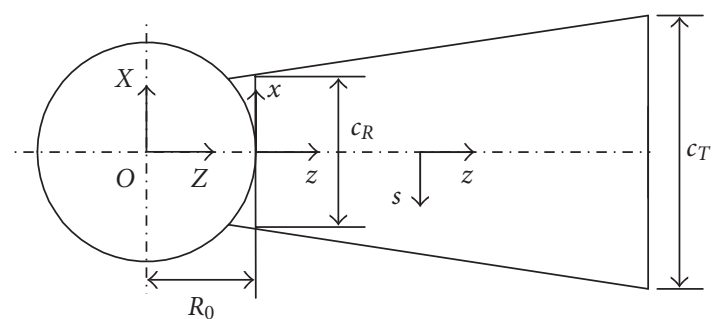

(a)

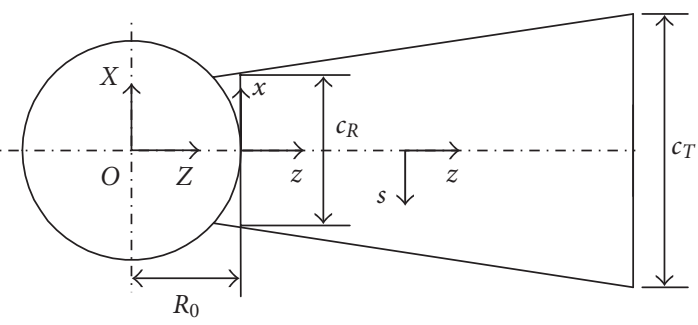

(b)

FIgURE 1: Geometric configuration of the rotating blade. Here $\sigma>1$.
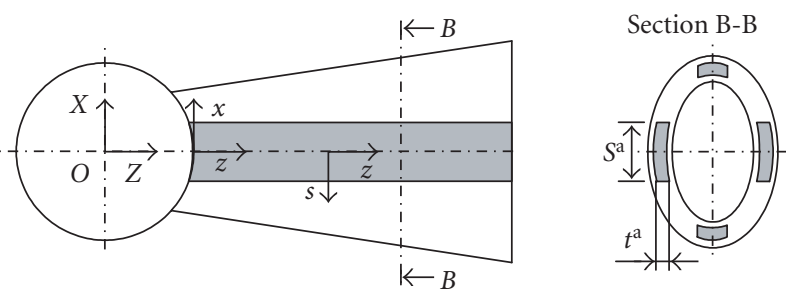

Figure 2: The distribution of piezoactuators.

height $b(\eta)$ of the midline cross-sectional profiles are represented as

$$
\left\{\begin{array}{l}
c(\eta) \\
b(\eta)
\end{array}\right\}=[1-\eta(1-\sigma)]\left\{\begin{array}{l}
c_{R} \\
b_{R}
\end{array}\right\}
$$

where $\sigma \equiv\left(c_{T} / c_{R}=b_{T} / b_{R}\right)$ defines the taper ratio, $0 \leq \sigma \leq 2$, where subscripts $T$ and $R$ identify the quantities at the beam tip and root, respectively, while $\eta(=z / L)$ is the dimensionless spanwise coordinate, $L$ being the beam span.

Moreover, one assumes that the beam is pretwisted and mounted on a rigid hub at the presetting angle $\gamma$, rotating with the constant angular velocity $\Omega$ as shown in Figure 3 .

The origin of the rotating system of coordinates $(x, y$, $z$ ) is located at an offset $R_{0}$ from the rotation axis. Besides this system of coordinates, we also define the local coordinates $\left(x^{p}, y^{p}, z^{p}\right)$, where $x^{p}$ and $y^{p}$ are the principal axes of an arbitrary beam cross-section. The two coordinate systems

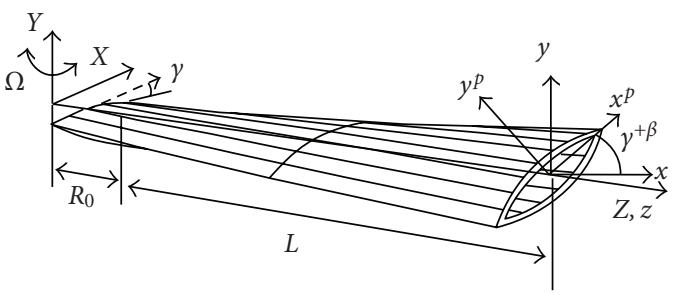

Figure 3: Geometry of the pretwisted beam.

are related by the following transformation relationships:

$$
\begin{gathered}
x=x^{p} \cos (\gamma+\beta(z))-y^{p} \sin (\gamma+\beta(z)), \\
y=x^{p} \sin (\gamma+\beta(z))+y^{p} \cos (\gamma+\beta(z)), \\
z=z^{p} .
\end{gathered}
$$

In (2), $\beta(z)=\beta_{0} z / L$ denotes the pretwist angle of a current beam cross-section; $\beta_{0}$ denotes the pretwist at the beam tip; (see Figure 3); $u, v$, and $w$ denote the components of the $3 \mathrm{D}$ displacement vector defined in terms of $1 \mathrm{D}$ generalized displacement measures (see Song et al. [3-5]). In the context of the present problem, a special case of plyangle distribution inducing the bending-bending elastic coupling will be applied. This is

$$
\theta(y)=\theta(-y), \quad \theta(x)=\theta(-x)
$$

The present plyangle configuration, referred to as the circumferentially uniform stiffness configuration, results in an exact decoupling of flapping-lagging-transverse shear motion from the extension-twist. Within this study, only the former elastic coupling will be considered.

In these conditions, the dynamic equations of pretwisted rotating blades in terms of displacement variables are expressed as

$$
\begin{aligned}
u(x, y, z, t)= & u_{0}(z, t) \\
v(x, y, z, t)= & v_{0}(z, t) \\
w(x, y, z, t)= & x(s) \theta_{y}(z, t)+y(s) \theta_{x}(z, t) \\
& +n\left[\frac{d y}{d s} \theta_{y}(z, t)-\frac{d x}{d s} \theta_{x}(z, t)\right] .
\end{aligned}
$$

In these equations, $u_{0}(z, t)$ and $v_{0}(z, t)$ denote the rigid body translations along the $x$ and $y$ axes, while $\theta_{x}(z, t)$ and $\theta_{y}(z, t)$ denote the rotations about the $x$ - and $y$-axes, respectively, and $(-h / 2 \leq n \leq h / 2)$ denotes the wall thickness coordinate, $h$ being the blade thickness. The expressions of $\theta_{x}$ and $\theta_{y}$ are

$$
\begin{aligned}
& \theta_{x}(z, t)=\gamma_{y z}(z, t)-v_{0}^{\prime}(z, t), \\
& \theta_{y}(z, t)=\gamma_{x z}(z, t)-u_{0}^{\prime}(z, t),
\end{aligned}
$$

where $\gamma_{x z}$ and $\gamma_{y z}$ are the transverse shear strains and the primes $(\square)^{\prime}$ denote the derivatives of $(\square)$ with respect to the spanwise coordinate $z$. The position vector of a point 
$M(x, y, z)$ belonging to the deformed structure is

$$
\mathbf{R}(x, y, z, t)=(x+u) \mathbf{i}+(y+v) \mathbf{j}+(z+w) \mathbf{k}+\mathbf{R}_{0},
$$

where $(\mathbf{i}, \mathbf{j}, \mathbf{k})$ are the unit vectors along the $x$-, $y$-, and $z$-axes, respectively.

Recalling that the spin rate is constant, with the help of equations expressing the time derivatives of unit vectors, one obtain the velocity and acceleration vectors of a point $M$ of the blade as

$$
\begin{aligned}
\dot{\mathbf{R}}= & V_{x} \mathbf{i}+V_{y} \mathbf{j}+V_{z} \mathbf{k}=\left(\dot{u}+\left(R_{0}+z+w\right) \Omega\right) \mathbf{i} \\
& +\dot{v} \mathbf{j}+(\dot{w}-(x+u) \Omega) \mathbf{k}, \\
\ddot{\mathbf{R}}= & a_{x} \mathbf{i}+a_{y} \mathbf{j}+a_{z} \mathbf{k}=\left(\ddot{u}+2 \dot{w} \Omega-(x+u) \Omega^{2}\right) \mathbf{i} \\
& +\ddot{v} \mathbf{j}+\left(\ddot{w}-2 \dot{u} \Omega-\left(R_{0}+z+w\right) \Omega^{2}\right) \mathbf{k} .
\end{aligned}
$$

\section{THE DYNAMIC EQUATIONS OF THE COMPOSITE BLADE}

Hamilton's principle is applied to obtain the equations of motion of adaptive rotating blades of nonuniform crosssection, and the associated boundary conditions. The variational principle may be stated as (Librescu [15])

$$
\begin{aligned}
\delta J=\int_{t_{0}}^{t_{1}} & {\left[\int_{\tau} \sigma_{i j} \delta \varepsilon_{i j} d \tau-\delta K\right.} \\
& \left.\quad-\int_{\Omega_{\sigma}} \underset{\sim}{s} \delta v_{i} d \Omega-\int_{\tau} \rho H_{i} \delta v_{i} d \tau\right] d t=0,
\end{aligned}
$$

where

$$
K=\frac{1}{2} \int_{\tau} \rho\left(\dot{\mathbf{R}}_{i} \cdot \dot{\mathbf{R}}_{i}\right) d \tau
$$

In these equations, $t_{0}$ and $t_{1}$ denote two arbitrary instants of time, $d \tau(\equiv d n d s d z)$ denotes the differential volume element, $H_{i}$ denote the components of the body forces, $\rho$ denotes the mass density, while $\delta$ denotes the variation operator. In light of (3), (4), (5), (6), and (7), upon imposing Hamilton's conditions $\delta v_{i}=0$ at $t_{0}$ and $t_{1}$; carrying out the integration over the circumferential and thickness coordinates, $s$ and $n$, respectively, one obtains for the variation of kinetic energy,

$$
\begin{aligned}
\int_{t_{0}}^{t_{1}} \delta T d t= & \int_{t_{0}}^{t_{1}} \int_{\tau} \rho(\dot{\mathbf{R}} \cdot \delta \ddot{\mathbf{R}}) d \tau d t \\
= & -\int_{t_{0}}^{t_{1}} d t \int_{\tau} \rho \ddot{\mathbf{R}} \cdot \delta \dot{\mathbf{R}} d \tau \\
=\int_{t_{0}}^{t_{1}} \int_{0}^{L}- & \left\{b_{1}\left(\ddot{u}_{0}+2 \dot{w}-\Omega-u_{0} \Omega^{2}\right) \delta u_{0}\right. \\
& +b_{1} \ddot{v}_{0} \delta v_{0}+I_{y y}\left(\ddot{\theta}_{y}-\Omega^{2} \theta_{y}\right) \delta \theta_{y} \\
& \left.+\left[I_{x x}\left(\ddot{\theta}_{x}-\Omega^{2} \theta_{x}\right)\right] \delta \theta_{x}\right\} d z d t .
\end{aligned}
$$

For the variation of the strain energy $\delta V$ one obtains

$$
\begin{gathered}
\int_{t_{0}}^{t_{1}} \delta V d t \\
=\int_{t_{0}}^{t_{1}} \int_{\tau} \sigma_{i j} \delta \varepsilon_{i j} d \tau d t \\
=\int_{0}^{L} \int_{c}\left[\mathrm{~N}_{z z} \delta \varepsilon_{z z}^{0}+L_{z z} \delta \varepsilon_{z z}^{n}+L_{s z} \delta \gamma_{s z}^{0}+L_{n z} \delta \gamma_{n z}\right] d s d z \\
=\int_{t_{0}}^{t_{1}}\left\{\int_{0}^{L}-\left\{\left(M_{y}^{\prime}-Q_{x}\right) \delta \theta_{y}+\left(M_{x}^{\prime}-Q_{y}\right) \delta \theta_{x}\right.\right. \\
\left.\quad+\left[Q_{x}^{\prime}+\left(T_{z} u_{0}^{\prime}\right)^{\prime}\right] \delta u_{0}+\left[Q_{y}^{\prime}+\left(T_{z} v_{0}^{\prime}\right)^{\prime}\right] \delta v_{0}\right\} d z \\
+\left[T_{z} \delta w_{0}+M_{y} \delta \theta_{y}+M_{x} \delta \theta_{x}\right. \\
\left.\left.+\left(Q_{x}+T_{z} u_{0}^{\prime}\right) \delta u_{0}+\left(Q_{y}+T_{z} v_{0}^{\prime}\right) \delta v_{0}\right]_{0}^{L}\right\} d t .
\end{gathered}
$$

These equations are represented in terms of $1 \mathrm{D}$ stress resultants and stress couples. Their expressions have been supplied by Song and Librescu [1], and will not be recorded here.

Reminding that in this case, an exact decoupling of flapping-lagging-transverse shear motion from the extensiontwist is obtained, the dynamic equations of pretwist rotating blades of nonuniform cross-section in terms of bending displacement variables are as (Song et al. [3], Na and Librescu $[12,13])$

$$
\begin{aligned}
\delta u_{0}: & {\left[a_{42}(z) \theta_{y}^{\prime}+a_{43}(z) \theta_{x}^{\prime}+a_{44}(z)\left(u_{0}^{\prime}+\theta_{y}\right)\right.} \\
& \left.+a_{45}(z)\left(v_{0}^{\prime}+\theta_{x}\right)\right]^{\prime} \\
& -b_{1} \ddot{u}_{0}+b_{1} u_{0} \Omega^{2}+b_{1} \Omega^{2}\left[R(z) u_{0}^{\prime}\right]^{\prime}+p_{x}=0, \\
\delta v_{0}: & {\left[a_{52}(z) \theta_{y}^{\prime}+a_{53}(z) \theta_{x}^{\prime}+a_{55}(z)\left(v_{0}^{\prime}+\theta_{x}\right)\right.} \\
& \left.+a_{54}(z)\left(u_{0}^{\prime}+\theta_{y}\right)\right]^{\prime} \\
& -b_{1} \ddot{v}_{0}+b_{1} \Omega^{2}\left[R(z) v_{0}^{\prime}\right]^{\prime}+p_{y}=0, \\
\delta \theta_{y}: & a_{22}(z) \theta_{y}^{\prime}+a_{23}(z) \theta_{x}^{\prime}+a_{24}(z)\left(u_{0}^{\prime}+\theta_{y}\right) \\
& \left.+a_{25}(z)\left(v_{0}^{\prime}+\theta_{x}\right)\right]^{\prime}-a_{44}(z)\left(u_{0}^{\prime}+\theta_{y}\right) \\
& -a_{43}(z) \theta_{x}^{\prime}-a_{45}(z)\left(v_{0}^{\prime}+\theta_{x}\right)-a_{42}(z) \theta_{y}^{\prime} \\
& -\left(b_{5}(z)+b_{15}(z)\right)\left(\ddot{\theta}_{y}-\Omega^{2} \theta_{y}\right)+m_{y}=0, \\
\delta \theta_{x}:[ & a_{33}(z) \theta_{x}^{\prime}+a_{32}(z) \theta_{y}^{\prime}+a_{34}(z)\left(u_{0}^{\prime}+\theta_{y}\right) \\
& \left.+a_{35}(z)\left(v_{0}^{\prime}+\theta_{x}\right)\right]^{\prime}-a_{55}(z)\left(v_{0}^{\prime}+\theta_{x}\right) \\
& -a_{52}(z) \theta_{y}^{\prime}-a_{54}(z)\left(u_{0}^{\prime}+\theta_{y}\right)-a_{53}(z) \theta_{x}^{\prime} \\
& -\left(b_{4}(z)+b_{14}(z)\right)\left(\ddot{\theta}_{x}-\Omega^{2} \theta_{x}\right)+m_{x}=0 .
\end{aligned}
$$

In these equations, see Oh et al. [16],

$$
R(z)=-\int_{L}^{z} b_{1}(z)(R+z) d z
$$

$a_{i j}(z)$ and $b_{i}(z)$ are stiffness and inertia terms supplied in Song and Librescu [1], while $p_{x}, p_{y}, m_{x}$, and $m_{y}$ are surface loads in the $x$ and $y$ directions and their moments about the respective axes. 
Assuming the blade to be clamped at $z=0$ and free at $z=L$, the boundary conditions are

at $z=0$ :

$$
u_{0}=0, \quad v_{0}=0, \quad \theta_{x}=0, \quad \theta_{y}=0
$$

and at $z=L$ :

$$
\begin{aligned}
\delta u_{0} & : a_{42}(L) \theta_{y}^{\prime}+a_{43}(L) \theta_{x}^{\prime}+a_{44}(L)\left(u_{0}^{\prime}+\theta_{y}\right) \\
& +a_{45}(L)\left(v_{0}^{\prime}+\theta_{x}\right)=0, \\
\delta v_{0}: & a_{52}(L) \theta_{y}^{\prime}+a_{53}(L) \theta_{x}^{\prime}+a_{55}(L)\left(v_{0}^{\prime}+\theta_{x}\right) \\
& +a_{54}(L)\left(u_{0}^{\prime}+\theta_{y}\right)=0, \\
\delta \theta_{y}: & a_{22}(L) \theta_{y}^{\prime}+a_{23}(L) \theta_{x}^{\prime}+a_{25}(L)\left(v_{0}^{\prime}+\theta_{x}\right) \\
& +a_{24}(L)\left(u_{0}^{\prime}+\theta_{y}\right)=\tilde{M}_{y}, \\
\delta \theta_{x} & : a_{33}(L) \theta_{x}^{\prime}+a_{32}(L) \theta_{y}^{\prime}+a_{35}(L)\left(v_{0}^{\prime}+\theta_{x}\right) \\
& +a_{34}(L)\left(u_{0}^{\prime}+\theta_{y}\right)=\tilde{M}_{x},
\end{aligned}
$$

where $\tilde{M}_{x}$ and $\tilde{M}_{y}$ denote the piezoelectrically induced boundary moments about $x$ - and $y$-axes (see Librescu et al. [8]).

\section{THE CONTROL LAW}

As it was shown in a number of previous works (see, e.g., Song et al. [3]) as a result of the distribution of piezoactuators along the entire beam span, and the application of an out-of-phase electrical current, piezoelectrically bending moments $\tilde{M}_{x}$ and $\tilde{M}_{y}$ are generated solely in the boundary conditions prescribed at the beam tip. These moments play the role of the boundary moments control. The adaptive nature of the rotating blade is introduced by requiring the applied electric field $\Sigma_{3}$ to be related to one of the mechanical quantities characterizing its dynamic response. Within the velocity feedback control law and depending on whether the control is associated with the lagging and flapping motions, the piezoelectrically induced bending moments at the blade tip, $\tilde{M}_{x}$ and $\tilde{M}_{y}$, are expressed as

$$
\begin{aligned}
& \tilde{M}_{x}(L)=K_{x} \dot{\theta}_{x}(L), \\
& \tilde{M}_{y}(L)=K_{y} \dot{\theta}_{y}(L),
\end{aligned}
$$

where $K_{x}$ and $K_{y}$ are dimensionless feedback control gains expressed in terms of the dimensional ones, $k_{x}$ and $k_{y}$, as

$$
\begin{aligned}
& K_{x}=k_{x}\left(\frac{L}{a_{33}^{0}}\right) \omega_{0}, \\
& K_{y}=k_{y}\left(\frac{L}{a_{22}^{0}}\right) \omega_{0},
\end{aligned}
$$

where $\omega_{0}$ is a reference natural frequency.

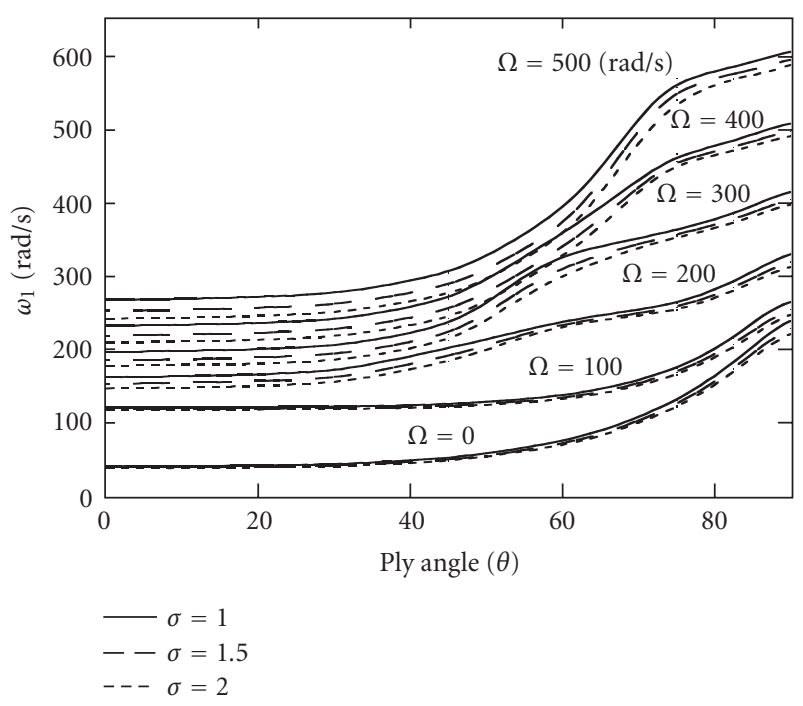

FIGURE 4: Variation of the first coupled flapping-lagging frequency versus plyangle for selected rotating speeds $\Omega(\mathrm{rad} / \mathrm{s})$, and three different taper ratios $(\gamma=\beta=0)$.

\section{NUMERICAL SIMULATIONS}

The numerical simulations are carried out for the blade characterized by (see also Figures 1 and 2)

$$
\begin{aligned}
& L=80 \mathrm{in}, \quad h=0.4 \mathrm{in}, \quad c_{R}=10 \mathrm{in}, \quad b_{R}=2.68 \mathrm{in}, \\
& t^{a}=0.00787 \mathrm{in}, \quad S^{a}=3.5 \mathrm{in}, \quad \bar{R}_{0}\left(\equiv \frac{R_{0}}{L}\right)=0.1,
\end{aligned}
$$

its material being of graphite/epoxy. In the on-axis configuration its properties are given by

$$
\begin{aligned}
E_{L} & =30 \times 10^{6} \mathrm{psi}, \quad E_{T}=0.75 \times 10^{6} \mathrm{psi}, \\
G_{L T} & =0.37 \times 10^{6} \mathrm{psi}, \quad G_{T T}=0.45 \times 10^{6} \mathrm{psi}, \\
\mu_{T T} & =\mu_{L T}=0.25, \quad \rho=14.3 \times 10^{-5} \mathrm{lbs} / \mathrm{in}^{4},
\end{aligned}
$$

where subscripts $L$ and $T$ denote directions parallel and transverse to the fibers, respectively. As concerns the characteristics of the piezoactuators, the ones madeup from PZT-4 piezoceramic whose properties are supplied by Berlincourt et al. [17] will be adopted also here. Herein the case of a step pressure pulse will be considered implying that

$$
p_{y}(s, z, t)\left(\equiv p_{y} H(t)\right)= \begin{cases}P_{m}, & t \geq 0 \\ 0, & t<0 .\end{cases}
$$

Throughout the results, unless otherwise specified, $P_{m}=$ $50 \mathrm{lb} / \mathrm{in}$.

Figures 4, 5, and 6 depict the variation of the first three coupled flapping-lagging frequencies as a function of the plyangle for selected rotational speeds $\Omega(\mathrm{rad} / \mathrm{s})$. The stiffening effect induced by the centrifugal forces is clearly revealed. The results shown in these plots highlight also sensitivity of modal eigenfrequencies to ply angle orientation of 


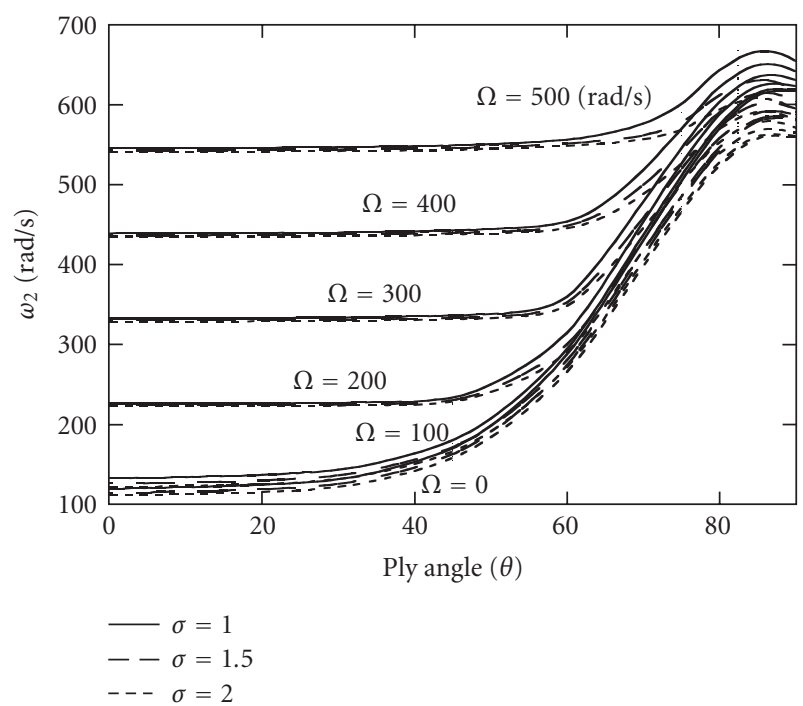

FIGURE 5: Variation of the second coupled flapping-lagging frequency versus plyangle for selected rotating speeds $\Omega(\mathrm{rad} / \mathrm{s})$, and three different taper ratios $(\gamma=\beta=0)$.

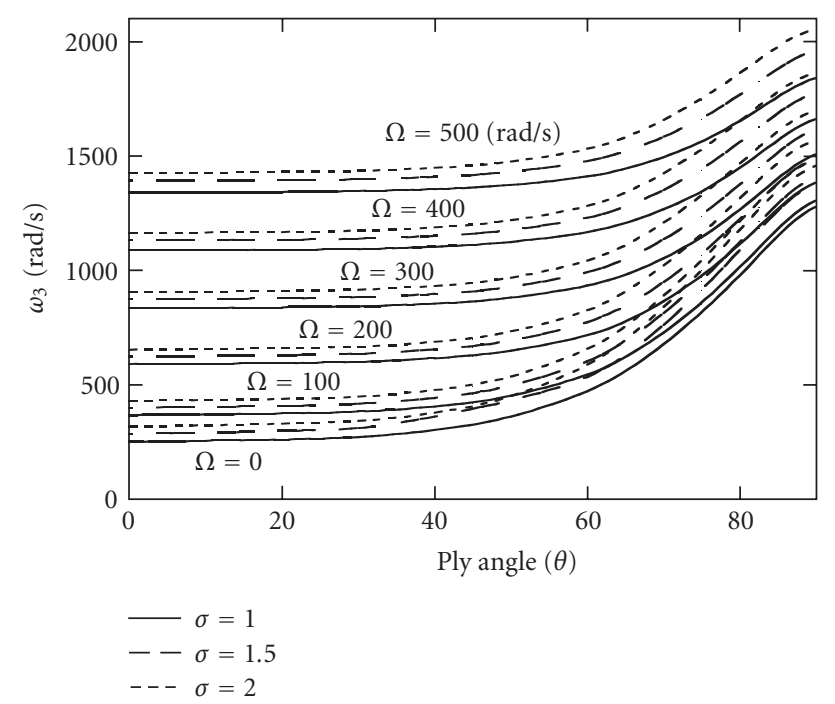

FIGURE 6: Variation of the third coupled flapping-lagging frequency versus plyangle for selected rotating speeds, and three different taper ratios $(\gamma=\beta=0)$.

the composite material. This provides a powerful tool in efforts of enhancing through structural tailoring the behavior of turbomachinery, fan, and helicopter blades for improved dynamic response. In these graphs, the influence of the taper ratio on coupled natural frequencies was also highlighted.

As in the case of nonrotating blades, the results reveal that, the beam taper plays a different role on the increase/ decrease of eigenfrequencies, this depends on the mode number (see Downs, [18] and Librescu and $\mathrm{Na}$ [9]).

Figures 7,8 , and 9 show the variation of the first three coupled natural frequencies as a function of the pretwist angle $\beta_{0}$ for selected rotational speeds and two taper ratios, in the case of the ply angle $\theta=90 \mathrm{deg}$. As in the previous figures,

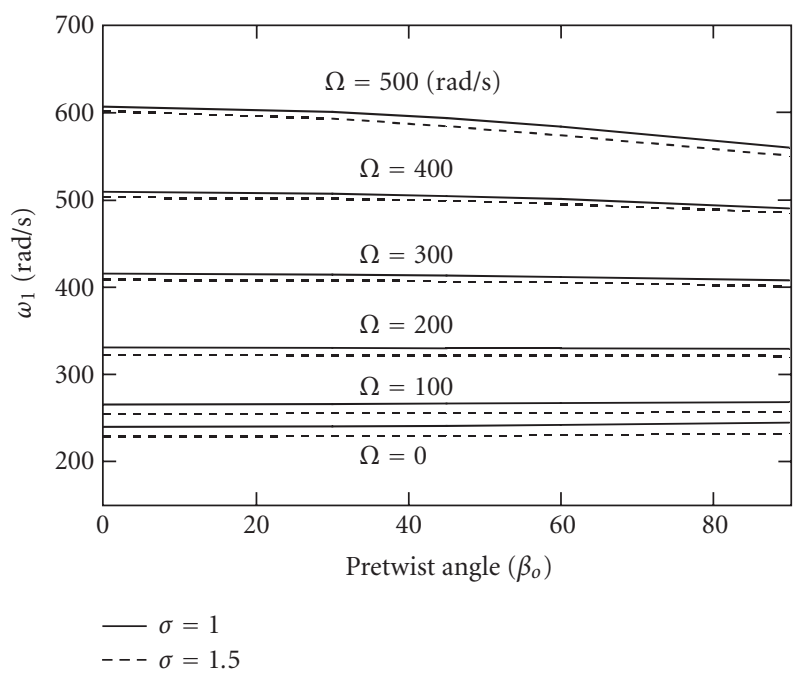

FIGURE 7: Variation of the first coupled frequency versus pretwisted angle for selected rotational speeds with two different taper ratios $(\theta=90 \mathrm{deg})$.

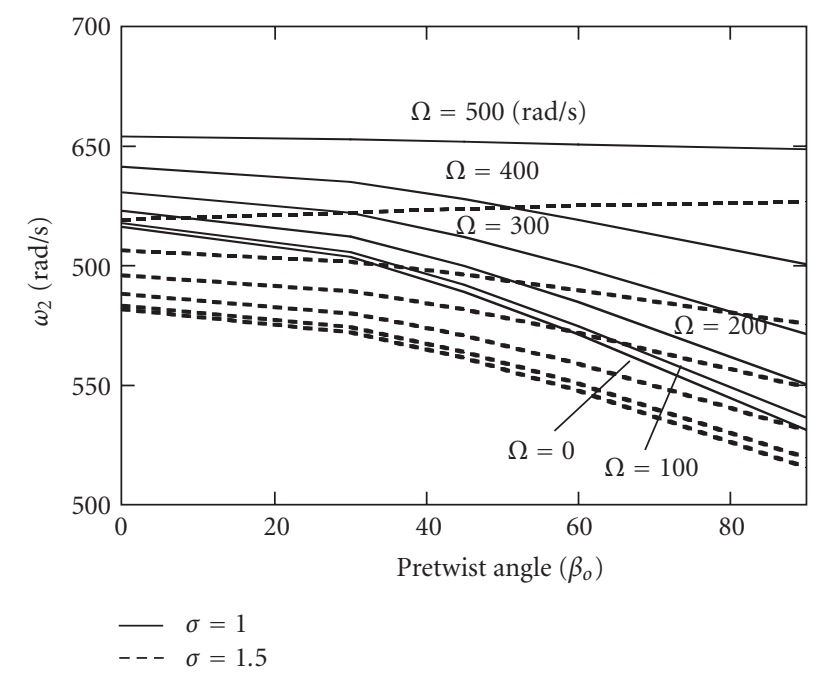

FIGURE 8: Variation of the second coupled frequency versus pretwisted angle for selected rotational speeds and two different taper ratios $(\theta=90 \mathrm{deg})$.

the important effect of the rotational speed on the coupled bending frequencies appears also from these figures.

The results in these figures are consistent with those obtained by Song and Librescu [2]. Figures 10 through 12 highlight the effects played by rotational speed and presetting angle on natural frequencies of the coupled bending-bending motion for selected ply angles, in the case of $\beta_{0}=45 \mathrm{deg}$. As it can be seen, for a nonrotating beam the effect of the presetting angle on the eigenfrequencies is immaterial.

For rotating beams, the character of the influence of the presetting angle depends on the mode number. As concerns the third coupled bending frequency, the trend is similar to the first frequency. 


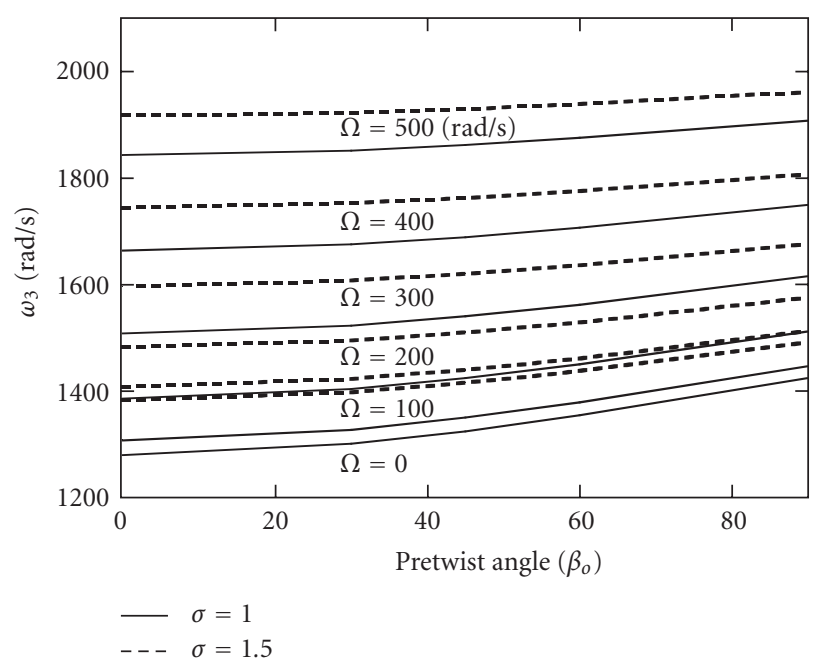

FIGURE 9: Variation of the third coupled frequency versus pretwisted angle for selected rotational speeds and two different taper ratios $(\theta=90 \mathrm{deg})$.

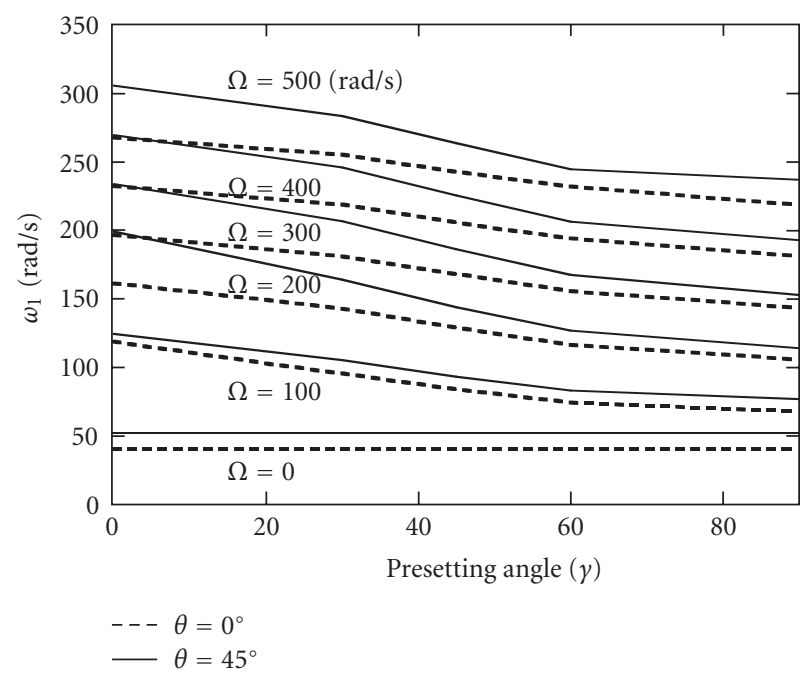

Figure 10: Variation of the first coupled frequency versus presetting angle for selected rotational speeds and two different ply angles ( $\left.\beta_{0}=45 \mathrm{deg}\right)$.

Figures 13, 14, and 15 show the variation of the open/ closed loop first three coupled natural frequencies as function of ply angle, for selected rotational speeds in the presence of the dimensionless velocity feedback gains $\left(K_{y}=K_{x}=\right.$ $\left.0.025, \gamma=\beta_{0}=0\right)$. The results reveal the important role played by the implementation of piezoelectric strain actuation on the eigenfrequencies. As concerns the implications of the ply angle, these are similar to those already considered in the case of inactivated rotating beams. From now on, the dynamic response of the rotating beam tip will be considered.

Herein, nondimensional flapping displacements $\bar{V}(\equiv$ $V(L) / L)$ and nondimensional lagging displacement $\bar{U}(\equiv$ $\left.U_{0}(L) / L\right)$ will be considered.

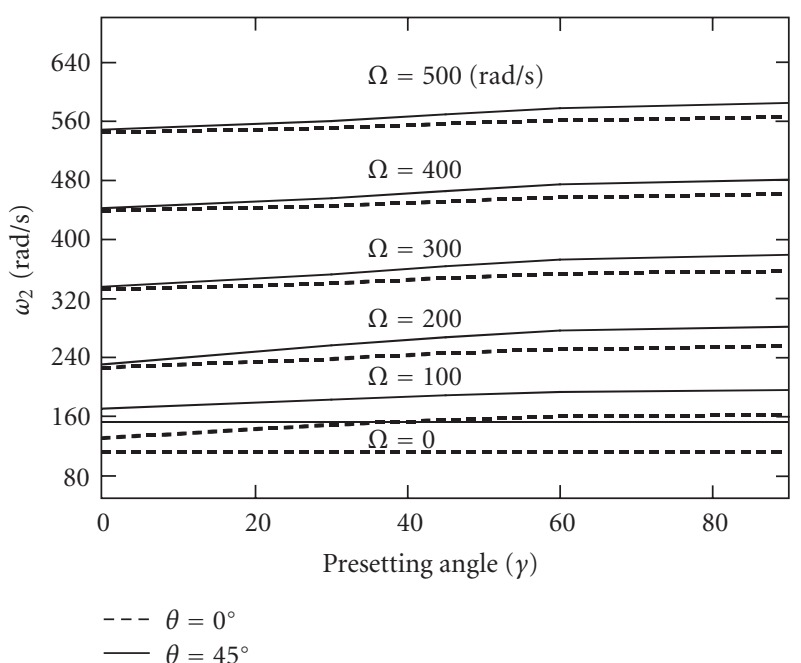

FIGURE 11: Variation of the second coupled frequency versus presetting angle for selected rotational speeds with two different ply angles $\left(\beta_{0}=45 \mathrm{deg}\right)$.

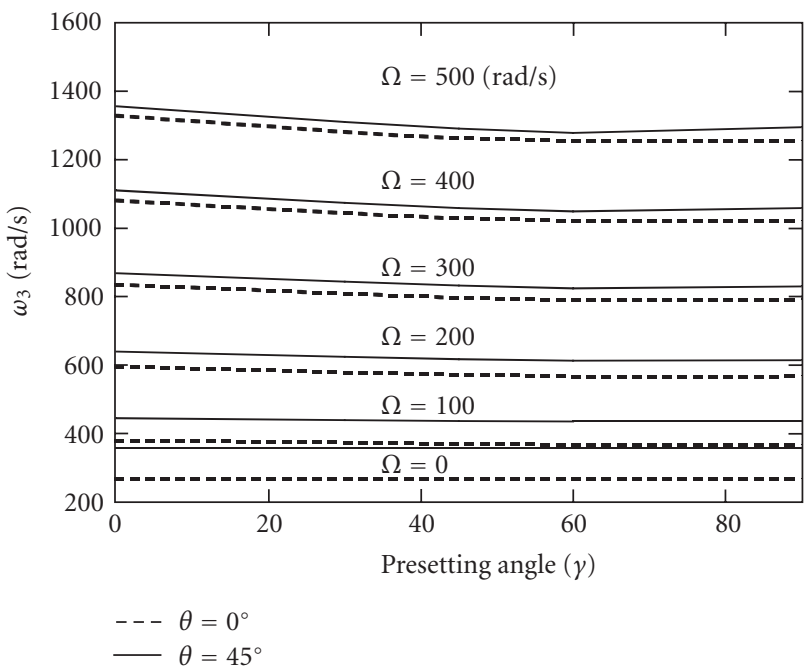

FIGURE 12: Variation of the third coupled frequency versus presetting angle for selected rotational speeds and two different ply angles $\left(\beta_{0}=45 \mathrm{deg}\right)$.

Figure 16 depicts the influence of the ply angle on the open/closed loop dimensionless flapping deflection timehistory of the blade subjected to a step pulse with $\sigma=1$, $\beta_{o}=\gamma=0$, and $\Omega=100 \mathrm{rad} / \mathrm{s}$, while Figure 17 highlights the influence of the ply angle on the open/closed loop dimensionless lagging deflection time-history of the blade tip, $\left(\sigma=1, \beta_{o}=\gamma=0\right.$, and $\left.\Omega=100 \mathrm{rad} / \mathrm{s}\right)$. In Figure 16, one obtains an attenuation of the dynamic response when using the larger ply angle, implying an increased flexural stiffness. Hence, the directional property of fiber reinforced composites can be used to effectively control the dynamic response of the structure. The results of Figure 17 depicting the lagging response to a step loading reflect a similar trend as in 


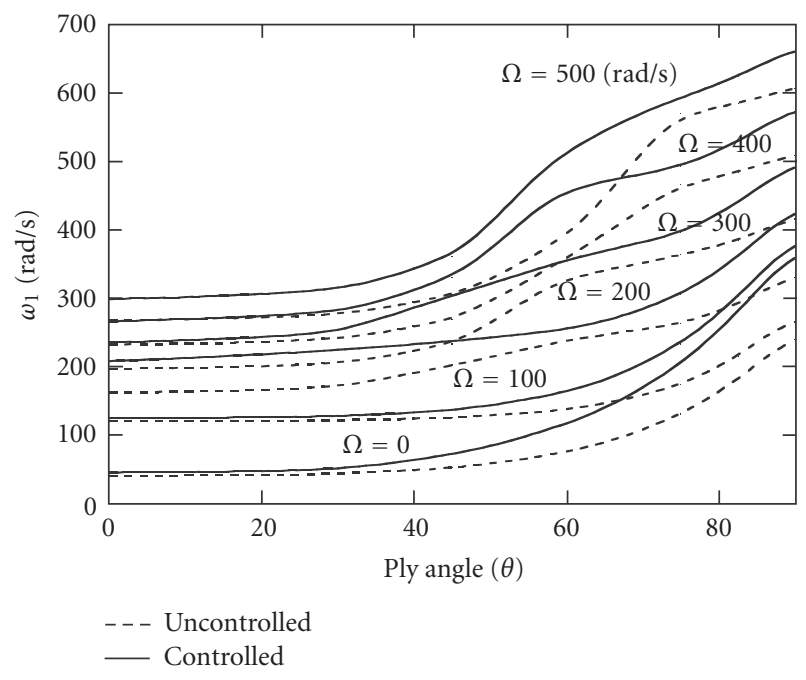

FIgURE 13: Variation of the first coupled open/closed flappinglagging frequency versus ply angle for selected rotating speeds with the piezoelectrically induced bending moment $\left(K_{y}=K_{x}=0.025\right.$, $\gamma=\beta=0)$.

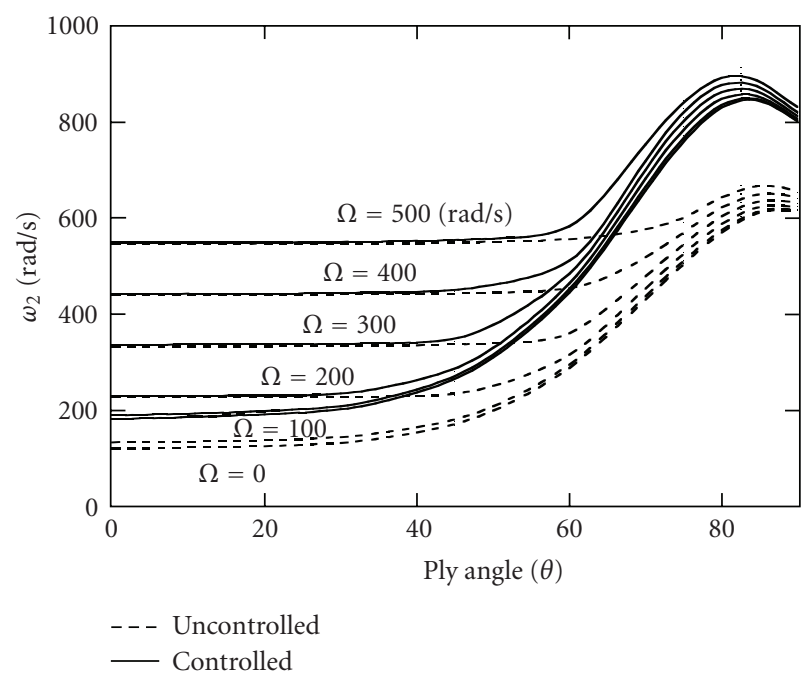

FIGURE 14: Variation of the second coupled open/closed flappinglagging frequency versus ply angle for selected rotating speeds with the piezoelectrically induced bending moments $\left(K_{y}=K_{x}=0.025\right.$, $\gamma=\beta=0$ ).

Figure 16. In spite of this, the active control appears to be much powerful in containing the oscillatory motion than the passive one as provided by the tailoring technique.

Figure 18 presents the combined influence of the rotating speed and of the piezoelectric strain actuation on flapping nondimensional deflection time-history of the beam, $(\sigma=1$, $\beta_{o}=\gamma=0$, and $\theta=60 \mathrm{deg}$.) The effect of the rotational speed on dynamic response of the blade exposed to a step pulse is due to the centrifugal stiffening that was well documented earlier. Moreover, the confinement of dynamic response of

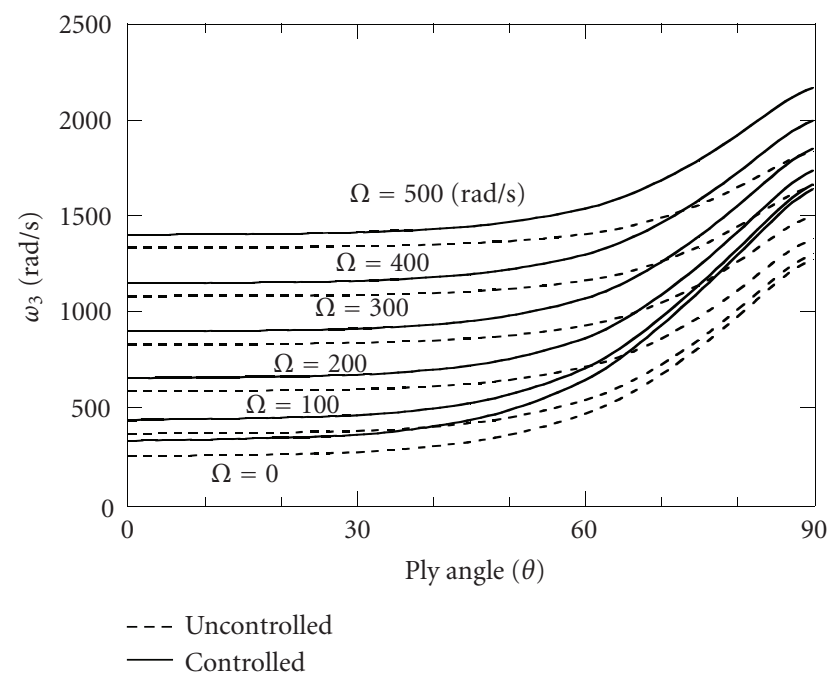

FIGURE 15: Variation of the third coupled open/closed flappinglagging frequency versus plyangle for selected rotating speeds with the piezoelectrically induced bending moments $\left(K_{y}=K_{x}=0.025\right.$, $\gamma=\beta=0)$.

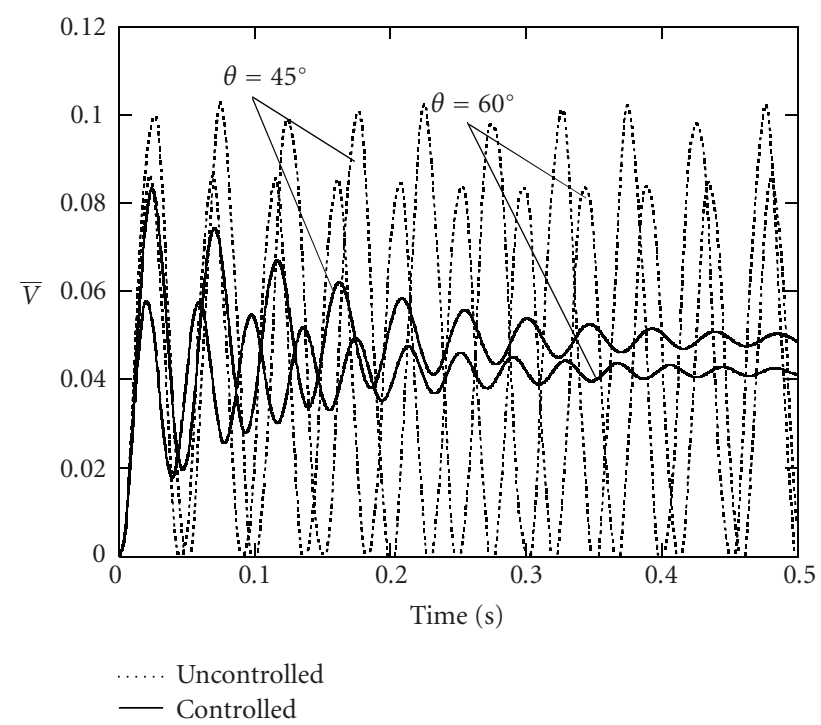

Figure 16: Influence of the ply angle on the uncontrolled/controlled nondimensional flapping deflection time-histories of the blade subjected to the step loading with $\sigma=1, \beta_{o}=\gamma=0$, and $\Omega=$ $100 \mathrm{rad} / \mathrm{s}$.

rotating beam is achieved via the piezoelectric strain actuation.

Figure 19 displays the influence of the pretwist angle on the open/closed loop flapping dimensionless deflection timehistory of a nonrotating blade exposed to a step pulse with $\sigma=1, \gamma=0, \Omega=0$, and $\theta=45 \mathrm{deg}$. As the pretwist angle increases, a marginal increase of the natural frequency is obtained. See in this sense also Song et al. [4]. A similar trend appears in the case of the rotating beam is shown in Figure 20. Figure 21 shows the effect of the presetting angle 


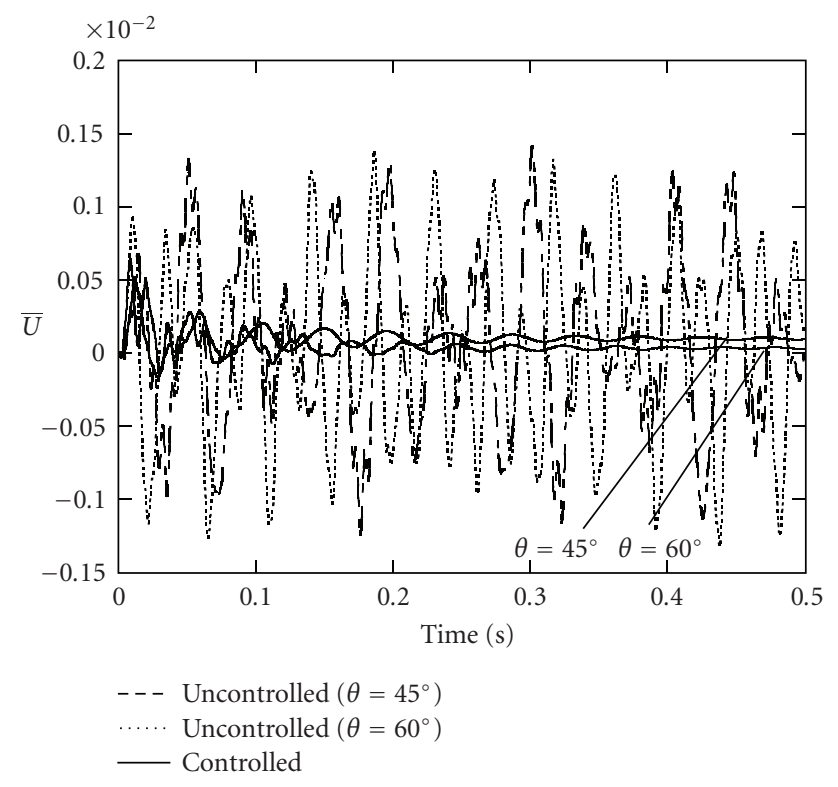

FIGURE 17: Influence of ply angle on the uncontrolled/controlled nondimensional lagging deflection time-history of the blade subjected to the step loading with $\sigma=1, \beta_{o}=\gamma=0$, and $\Omega=100 \mathrm{rad} / \mathrm{s}$.

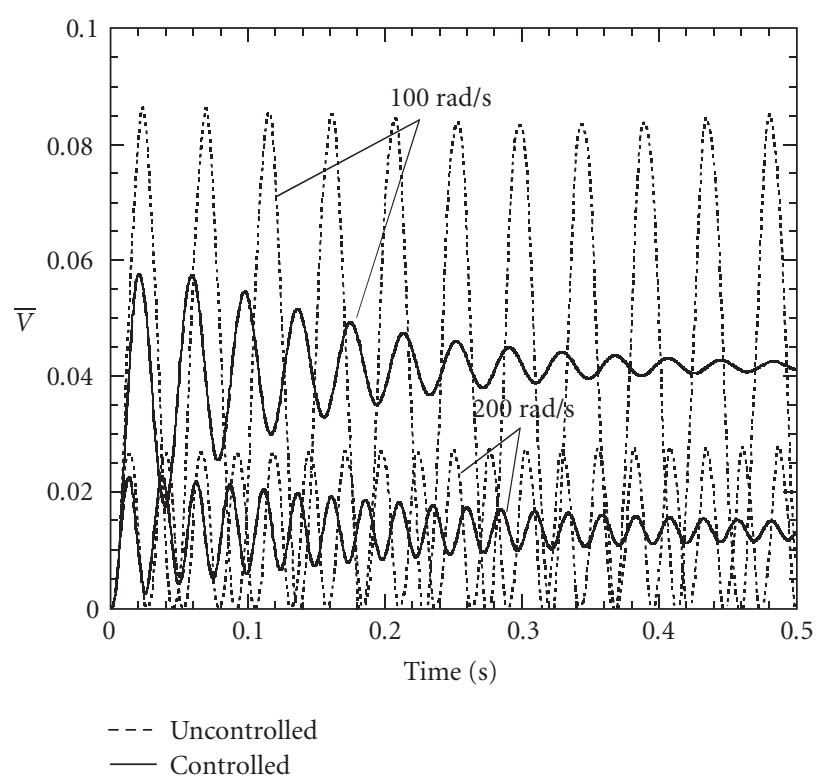

FIGURE 18: Influence of the rotating speed on the uncontrolled/controlled flapping nondimensional deflection time-history to the step loading with $\sigma=1, \beta_{o}=\gamma=0$, and $\theta=60 \mathrm{deg}$.

on the uncontrolled/controlled dimensionless flapping deflection time-history of the blade subjected to a step pulse, when $\sigma=1, \beta_{o}=0, \Omega=0 \mathrm{rad} / \mathrm{s}$, and $\theta=45 \mathrm{deg}$. The results reveal that for selected presetting angles, the dynamic response amplitudes coincide with that reported, for example, in the papers by $\mathrm{Oh}$ et al. [16].

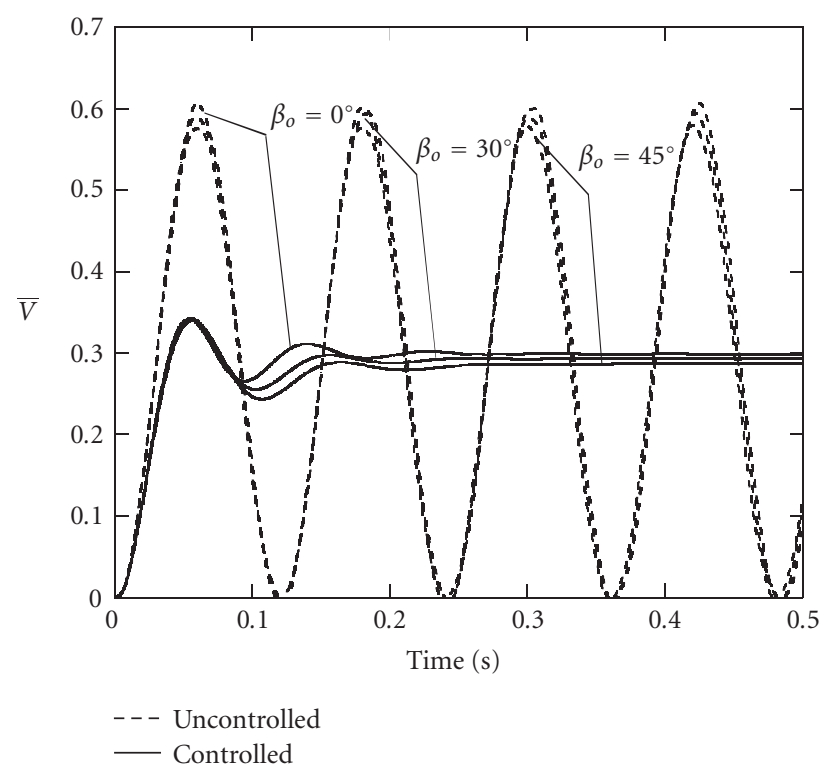

FIGURE 19: Influence of the pretwisted angle on the uncontrolled/ controlled flapping nondimensional deflection time-history of the nonrotating blade subjected to a step pulse with $\sigma=1, \gamma=0$ deg, $\Omega=0 \mathrm{rad} / \mathrm{s}$, and $\theta=45 \mathrm{deg}$.

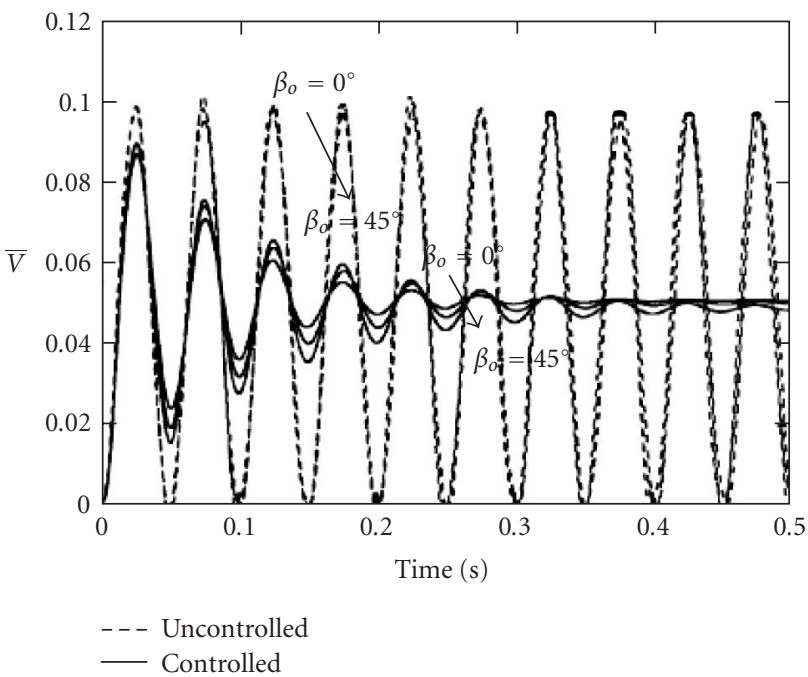

FIGURE 20: Influence of the pretwisted angle on the uncontrolled/ controlled flapping nondimensional deflection time-history of rotating blade subjected to the step loading with $\sigma=1, \gamma=0 \mathrm{deg}$, $\Omega=100 \mathrm{rad} / \mathrm{s}$, and $\theta=45 \mathrm{deg}$.

\section{CONCLUSIONS}

A dynamic structural model of nonuniform rotating composite blades and incorporating the pretwist and presetting angles, as well as the anisotropy of constituent materials was presented. In addition, based on the converse piezoelectric 


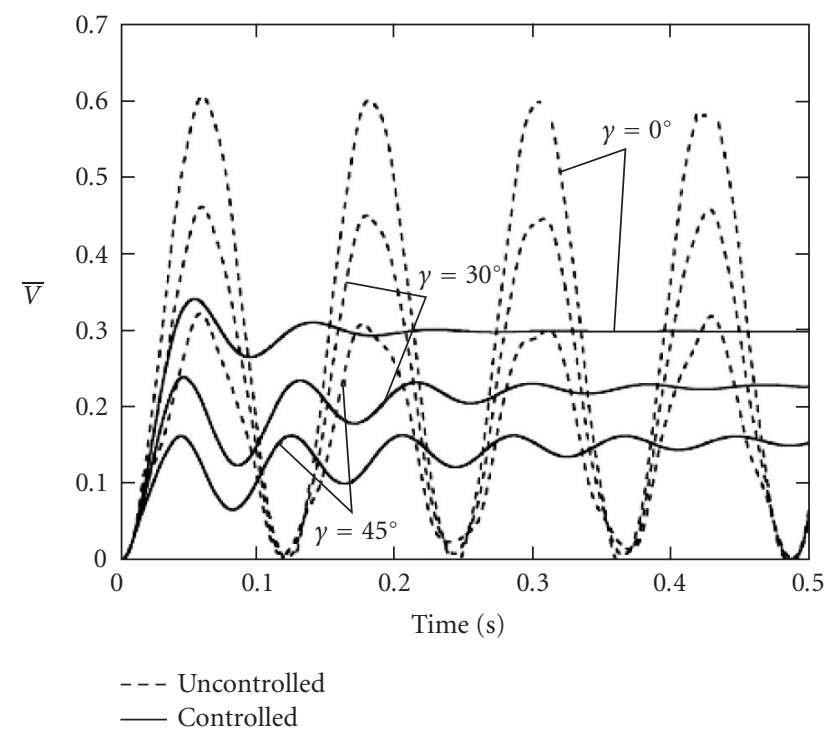

Figure 21: Influence of the presetting angle on uncontrolled/controlled nondimensional flapping deflection time-history of the blade subjected to the step loading with $\sigma=1, \beta_{o}=0, \Omega=0$, and $\theta=45 \mathrm{deg}$.

effect, a distributed control methodology based on piezoelectric strain actuation in conjunction with the velocity feedback control law aimed at controlling the flapping and lagging dynamic response of rotating blades was developed. The strong effect played by the directionality property of advanced composite materials and of the piezoelectric actuation on their free and dynamic response characteristics was highlighted. The results presented here are likely to provide valuable information to the engineers involved in the design of advanced turbomachinery rotating blades.

\section{REFERENCES}

[1] O. Song and L. Librescu, "Free vibration of anisotropic composite thin-walled beams of closed cross-section contour," Journal of Sound and Vibration, vol. 167, no. 1, pp. 129-147, 1993.

[2] O. Song and L. Librescu, "Structural modeling and free vibration analysis of rotating composite thin-walled beams," Journal of the American Helicopter Society, vol. 42, no. 4, pp. 358369, 1997.

[3] O. Song, L. Librescu, and S.-Y. Oh, "Vibration of pretwisted adaptive rotating blades modeled as anisotropic thin-walled beams," AIAA Journal, vol. 39, no. 2, pp. 285-295, 2001.

[4] O. Song, L. Librescu, and S.-Y. Oh, "Dynamics of pretwisted rotating thin-walled beams operating in a temperature environment," Journal of Thermal Stresses, vol. 24, no. 3, pp. 255279, 2001.

[5] O. Song, S.-Y. Oh, and L. Librescu, "Dynamic behavior of elastically tailored rotating blades modeled as pretwisted thinwalled beams and incorporating adaptive capabilities," International Journal of Rotating Machinery, vol. 8, no. 1, pp. 13-25, 2002.

[6] S.-Y. Oh, L. Librescu, and O. Song, "Thermoelastic modeling and vibration of functionally graded thin-walled rotating blades," AIAA Journal, vol. 41, no. 10, pp. 2051-2061, 2003.
[7] S.-Y. Oh, L. Librescu, and O. Song, "Vibration of turbomachinery rotating blades made-up of functionally graded materials and operating in a high temperature field," Acta Mechanica, vol. 166, no. 2, pp. 69-87, 2003.

[8] L. Librescu, O. Song, and C. A. Rogers, "Adaptive vibrational behavior of cantilevered structures modeled as composite thin-walled beams," International Journal of Engineering Science, vol. 31, no. 5, pp. 775-792, 1993.

[9] L. Librescu and S. S. Na, "Active vibration control of doubly tapered thin-walled beams using piezoelectric actuation," ThinWalled Structures, vol. 39, no. 1, pp. 65-82, 2001.

[10] S. S. Na and L. Librescu, "Dynamic behavior of aircraft wings modeled as doubly-tapered composite thin-walled beams," in Recent Advances in Solids and Structures, Y. W. Kwon and H. H. Chung, Eds., vol. 398 of Pressure Vessels \& Piping Division, PVP, pp. 59-68, American Society of Mechanical Engineers, New York, NY, USA, 1999.

[11] S. S. Na and L. Librescu, "Modeling and vibration feedback control of rotating tapered beams incorporating adaptive capabilities," in Recent Advances in Solid and Structures, vol. 415 of Pressure Vessels \& Piping Division, PVP, pp. 35-43, American Society of Mechanical Engineers, New York, NY, USA, 2000 .

[12] S. S. Na and L. Librescu, "Dynamic response of elastically tailored adaptive cantilevers of nonuniform cross section exposed to blast pressure pulses," International Journal of Impact Engineering, vol. 25, no. 9, pp. 847-867, 2001.

[13] S. S. Na and L. Librescu, "Optimal dynamic response control of elastically tailored nonuniform thin-walled adaptive beams," Journal of Aircraft, vol. 39, no. 3, pp. 469-479, 2002.

[14] S. S. Na, L. Librescu, and J.-H. Shim, "Modified bang-bang vibration control applied to adaptive thin-walled beam cantilevers," AIAA Journal, vol. 42, no. 8, pp. 1717-1721, 2004.

[15] L. Librescu, Elastostatics and Kinetics of Anisotropic and Heterogeneous Shell-Type Structures, Noordhoff, Leyden, The Netherlands, 1975.

[16] S.-Y. Oh, O. Song, and L. Librescu, "Effects of pretwist and presetting on coupled bending vibrations of rotating thinwalled composite beams," International Journal of Solids and Structures, vol. 40, no. 5, pp. 1203-1224, 2003.

[17] D. A. Berlincourt, D. R. Curran, and H. Jaffe, "Piezoelectric and piezomagnetic materials and their functions as transducers," in Physical Acoustics: Principles and Methods, Vol. 1, Part A, W. P. Mason, Ed., pp. 202-204, Academic Press, New York, NY, USA, 1964.

[18] B. Downs, "Transverse vibration of cantilever beams having unequal breadth and depth tapers," ASME Transactions: Journal of Applied Mechanics, vol. 44, no. 4, pp. 737-742, 1977. 

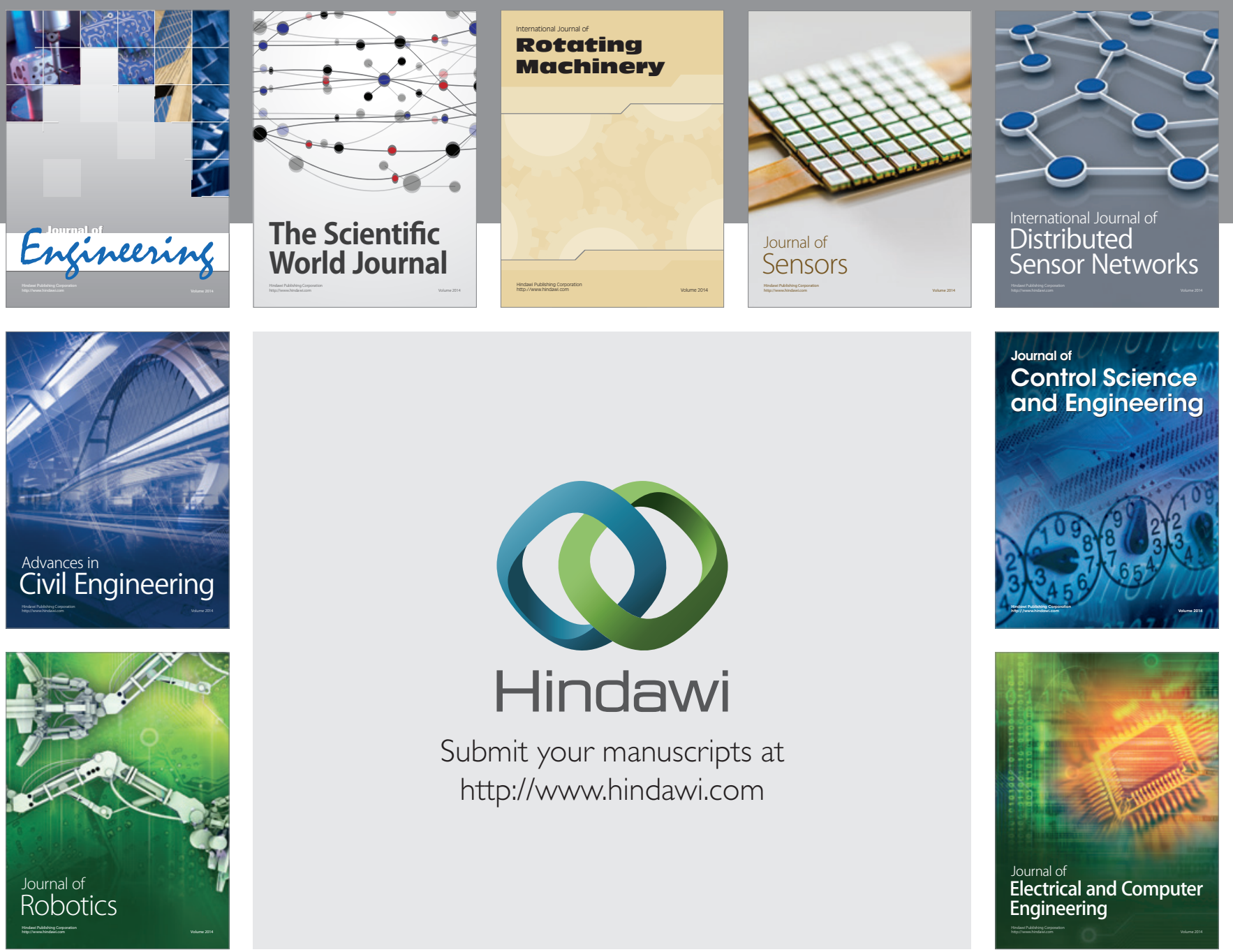

Submit your manuscripts at

http://www.hindawi.com
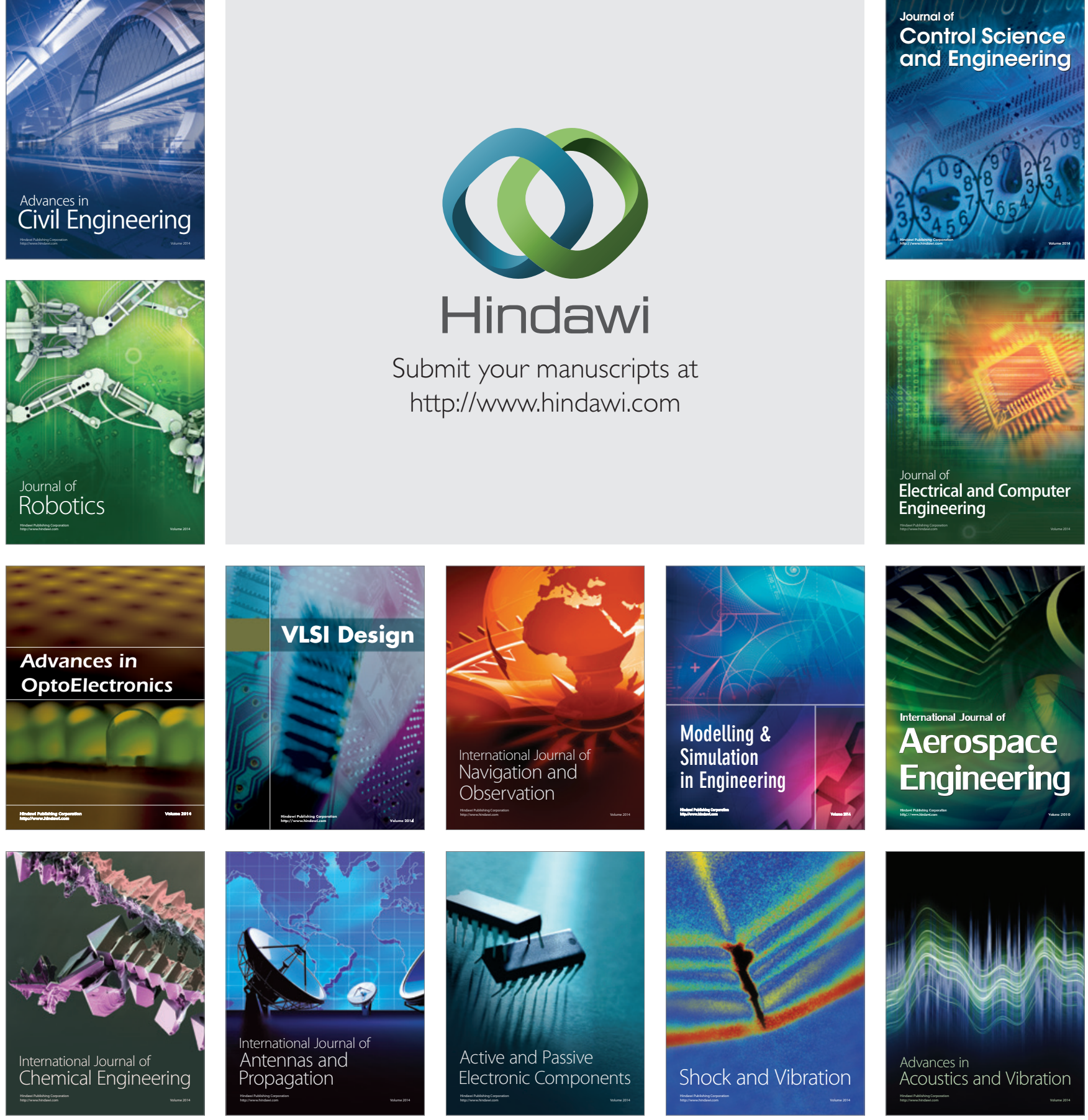